
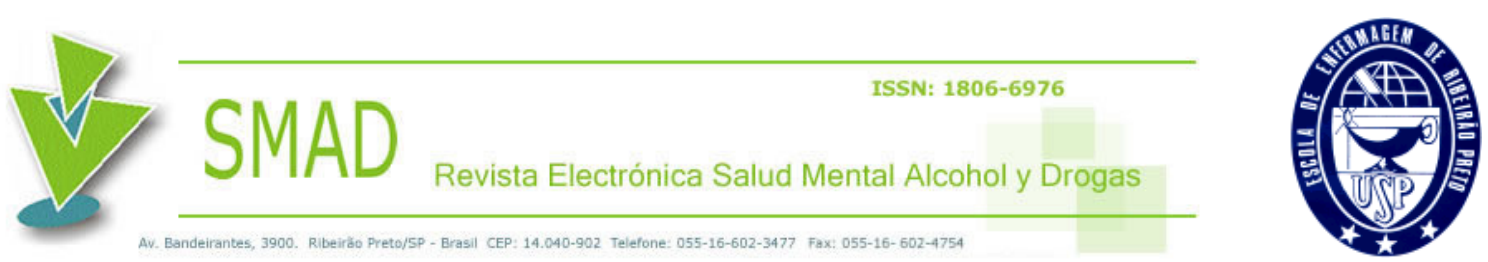

\title{
CONFIABILIDADE DO TESTE DE IDENTIFICAÇÃO DE TRANSTORNOS DEVIDO AO USO DE ÁLCOOL (AUDIT) EM ADOLESCENTES
}

\section{Fernanda Pavarina Mattara ${ }^{1}$; Priscila Milene Ângelo ${ }^{2}$; João Bosco Faria ${ }^{3}$; Juliana Alvares Duarte Bonini Campos ${ }^{4}$}

O objetivo deste trabalho foi estimar a confiabilidade do teste de identificação de transtornos devido ao uso de álcool (AUDIT), aplicado em adolescentes. Foram avaliados 141 estudantes com idade de 14 a 17 anos $(14,93 \pm 0,62)$. A consistência interna do instrumento foi estimada pelo coeficiente alfa de Cronbach e sua reprodutibilidade pela estatística Kappa ponderada por ponto e por intervalo de $95 \%$ de confiança. O AUDIT apresentou excelente consistência interna $\left(\alpha=0,80, \mathrm{r}_{\text {inter-item }}=0,29\right)$. A maioria das questões apresenta reprodutibilidade classificada como "boa". Pode-se concluir que o AUDIT revelou-se instrumento confiável para o levantamento de informações sobre o consumo de álcool em adolescentes.

Descritores: Alcoolismo; Adolescente; Reprodutividade dos Testes.

\section{RELIABILITY OF THE ALCOHOL USE DISORDERS IDENTIFICATION TEST (AUDIT) IN ADOLESCENTS}

The objective of this study was to estimate the reliability of the alcohol use disorders identification test (AUDIT) applied to adolescents. Evaluations were performed with 141 students of ages ranging from 14 to 17 years $(14.93 \pm 0.62)$. The internal consistency of the instrument was estimated by Cronbach's alpha coefficient and its reproducibility by weighted Kappa statistics by score and by a reliability interval of $95 \%$. The AUDIT presented excellent internal consistency $\left(\alpha=0.80, r_{\text {inter-item }}=0.29\right)$. Most questions present reproducibility ranked as "good". It is concluded that the AUDIT is a reliable instrument to survey information about alcohol use in adolescents.

Descriptors: Alcoholism; Adolescente; Reproducibility of Results.

1-Nutricionista, Mestranda do Programa de Pós-Graduação em Alimentos e Nutrição da Faculdade de Ciências Farmacêuticas da Universidade Estadual Paulista "Júlio de Mesquita Filho". E-mail: fermattara@terra.com.br

2-Nutricionista, Doutoranda do Programa de Pós-Graduação em Alimentos e Nutrição da Faculdade de Ciências Farmacêuticas da Universidade Estadual Paulista "Júlio de Mesquita Filho". E-mail: pmileneangelo@yahoo.com.br

3-Químico, Professor Adjunto do Departamento de Alimentos e Nutrição da Faculdade de Ciências Farmacêuticas da Universidade Estadual Paulista "Júlio de Mesquita Filho". E-mail: fariajb@fcfar.unesp.br 4-Dentista, Professor Doutor do Departamento de Odontologia Social da Faculdade de Odontologia de Araraquara da Universidade Estadual Paulista “Júlio de Mesquita Filho". E-mail: jucampos@ foar.unesp.br

Autor Correspondente: Juliana Alvares Duarte Bonini Campos Endereço para Correspondência: Universidade Estadual Paulista "Júlio de Mesquita Filho", Faculdade de Odontologia de Araraquara, Departamento de Odontologia Social. Rua Humaitá, 1680, Bairro Centro, CEP 14801-903, Araraquara, SP, Brasil. Telefone: +55 (16) 3301-6358. Fax: +55 (16) 3301-6343. E-mail: jucampos@ foar.unesp.br

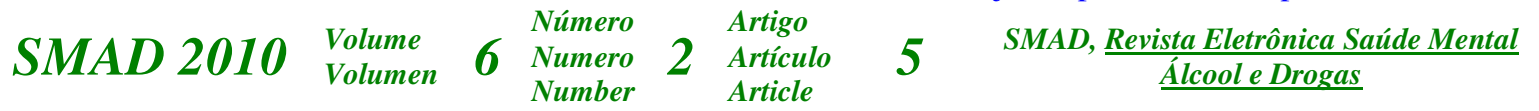




\section{CONFIABILIDAD DEL TEST DE IDENTIFICACIÓN DE TRANSTORNOS DEBIDOS AL USO DE ALCOHOL (AUDIT) EN ADOLESCENTES}

El objetivo de este trabajo fue estimar la confiabilidad del Test de identificación de transtornos debidos al uso de alcohol (AUDIT) aplicado en adolescentes. Fueron evaluados 141 estudiantes con edades entre 14 y 17 años $(14,93 \pm 0,62)$. La consistencia interna del instrumento fue estimada por el Coeficiente alfa-Cronbach, y su reproductibilidad por la estadística Kappa ponderada por punto y por intervalo de $95 \%$ de confianza. El AUDIT exhibió excelente consistencia interna $\left(\alpha=0,80, \mathrm{r}_{\text {inter-item }}=0,29\right)$. La mayoría de las cuestiones demuestra una reproductibilidad clasificada como "buena". Se puede concluir en que el AUDIT demuestra ser un instrumento confiable para la recopilación de informaciones sobre el consumo de alcohol en adolescentes.

Descriptores: Alcoholismo; Adolescente; Reproducibilidad de Resultados. 

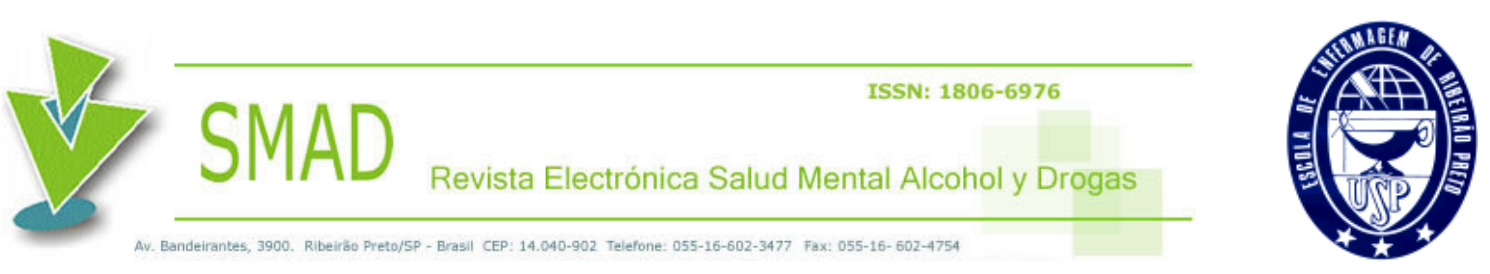

\section{Introdução}

O uso indevido de bebidas alcoólicas é considerado grave problema de saúde pública que atinge a população adulta e adolescente, acarretando sérias consequências na saúde física e mental da população ${ }^{(1)}$. Diferentes estudos, nacionais e internacionais, sistematicamente, confirmam que, se o álcool é facilmente obtido e fartamente propagandeado, isso se reflete em seu consumo precoce e disseminado ${ }^{(2)}$.

Em levantamento realizado com amostra de adolescentes representativa da população de Porto Alegre, RS, os autores verificaram que $71 \%$ dos 950 jovens participantes, de 10 a 18 anos, já haviam experimentado bebidas alcoólicas ${ }^{(3)}$.

De acordo com os resultados do levantamento realizado pelo Centro Brasileiro de Informações sobre Drogas Psicotrópicas (CEBRID) ${ }^{(4)}$, quanto ao consumo de álcool em estudantes do ensino fundamental e médio da rede pública de ensino, em 27 capitais brasileiras, com a participação de 48.155 estudantes, 65,20\% dos jovens consumiam bebidas alcoólicas e $6,70 \%$ podiam ser classificados como dependentes. Em média, o

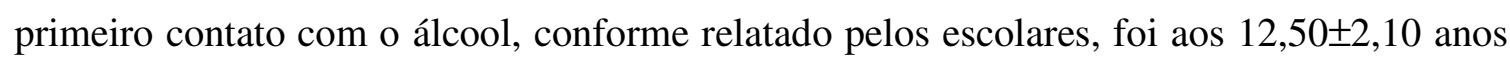
de idade, apontando para a precocidade do consumo de bebidas alcoólicas.

Os adolescentes são, em todo o mundo, o grupo populacional que apresenta os maiores riscos em relação ao consumo de bebidas alcoólicas, o que aponta para a necessidade de rastreamento e monitoramento do padrão de beber, a fim de prevenir e tratar SMAD $2010 \begin{aligned} & \begin{array}{l}\text { Volume } \\ \text { Volumen }\end{array} \\ & \text { Solumero }\end{aligned} \quad \begin{aligned} & \begin{array}{l}\text { Número } \\ \text { Number } \\ \text { Number }\end{array} \\ & 2\end{aligned} \begin{aligned} & \begin{array}{l}\text { Artigo } \\ \text { Artículo } \\ \text { Article }\end{array} \\ & \text { SMAD, Revista Eletrônica Saúde Mental }\end{aligned}$ 

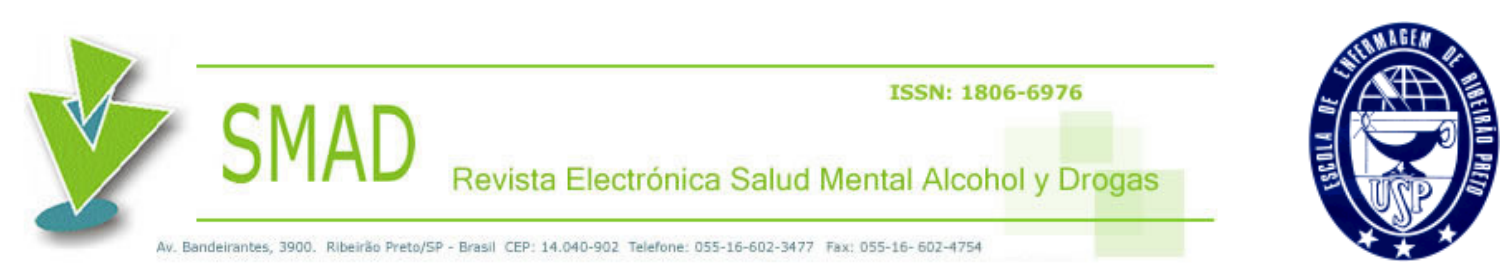

os transtornos decorrentes do uso de bebidas alcoólicas ${ }^{(5)}$.

Entre os instrumentos de rastreamento, pode-se destacar o teste de identificação de transtornos devido ao álcool (AUDIT), composto por 10 questões de autorrelato, formulado em escala Likert, que foi desenvolvido para avaliar o consumo de álcool nocivo, ou de risco, recomendado pela Organização Mundial de Saúde ${ }^{(5-6)}$.

O AUDIT foi desenvolvido e avaliado por período de duas décadas em um projeto colaborativo entre seis países (Austrália, Bulgária, Quênia, México, Noruega e Estados Unidos), com o objetivo de atender às diferentes realidades socioculturais e econômicas ${ }^{(7-9)}$. É instrumento amplamente utilizado em âmbito nacional e internacional para avaliar grupos populacionais ou indivíduos, quanto ao padrão do uso de álcool, identificando aqueles que necessitam de níveis diferenciados de intervenção. Considerando-se a importância desse instrumento, cabe ressaltar a importância de se realizar preliminarmente o estudo de confiabilidade das informações obtidas junto à população de estudo, visando assegurar a qualidade da informação aferida.

O AUDIT, quando comparado a outros 22 instrumentos, é aquele que apresenta as características psicométricas mais sofisticadas com fidedignidade e validade estimadas em várias populações de diversos países ${ }^{(7-23)}$.

Considerando, portanto, a importância de se obter dados de qualidade e as dificuldades encontradas na abordagem dos adolescentes, este estudo foi realizado com o objetivo de estimar a confiabilidade do teste de identificação de transtornos devido ao álcool (AUDIT), quando aplicado em adolescentes.

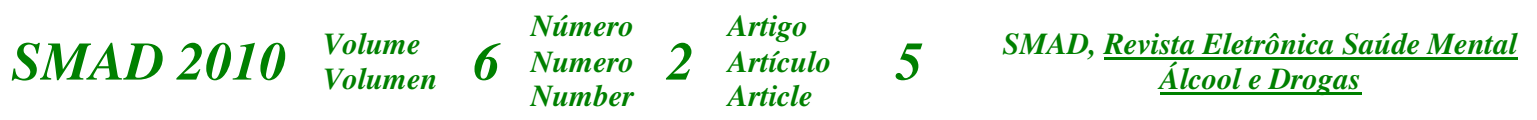




\section{Material e Métodos}

Participaram deste estudo, 141 estudantes de ambos os sexos, matriculados no $1^{\circ}$ ano do ensino médio, em 2009, de uma instituição da rede pública do município de Araraquara, SP, sendo adotado delineamento amostral não probabilístico. Os questionários foram aplicados em sala de aula, em horário previamente agendado com a direção da escola, em dois momentos distintos, com intervalo de 7 dias. Receberam os questionários apenas os alunos cujos pais preencheram e concordaram com o termo de consentimento livre e esclarecido, e os adolescentes consentiram com o preenchimento sério e comprometido do AUDIT, solicitado em visitas de esclarecimento, realizadas pelo pesquisador em momento anterior.

Para a caracterização da amostra, foram obtidas informações como idade, sexo, número de pessoas residentes no domicílio, presença ou não de religião, prática de esportes e trabalho. Em relação aos pais, os alunos foram questionados quanto ao consumo de bebidas alcoólicas dos mesmos e sobre o relacionamento familiar.

A prática de esportes, o trabalho, religião e o consumo de bebidas alcoólicas pelos pais foram avaliados em escala dicotômica e, para o relacionamento familiar, foram realizadas cinco opções de resposta. O nível econômico e de escolaridade foram estimados, segundo o Critério Brasil $2008^{(24)}$.

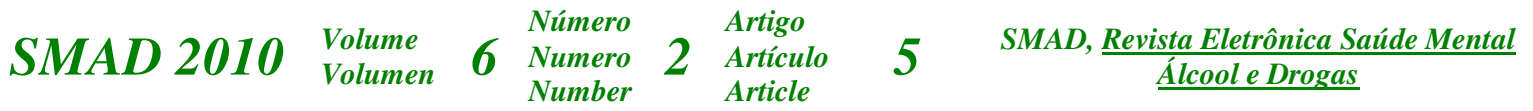




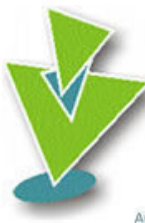

Para identificação de desordens, devido ao álcool, utilizou-se o questionário AUDIT já validado em português ${ }^{(25-26)}$. Esse instrumento, composto por 10 questões objetivas, permite respostas com pesos preestabelecidos, variando de 0 a 4 . $\mathrm{O}$ somatório do peso de cada questão indica a classificação de cada indivíduo frente ao consumo de bebidas alcoólicas, sendo de 0 a 7 classificado como beber moderado, de 8 a 15 padrão de beber de risco, de 16 a 19 uso nocivo de álcool e de 20 a 40 indica possível dependência de álcool ${ }^{(5)}$.

A consistência interna do AUDIT foi estimada no primeiro momento de preenchimento do AUDIT pelo coeficiente $\alpha-$ Cronbach $^{(27-28)}$. Para o estudo da reprodutibilidade intraexaminador, utilizou-se a estatística Kappa com ponderação linear $\left(\kappa_{\mathrm{p}}\right)$ por ponto e por intervalo de $95 \%$ de confiança, sendo adotado o nível de significância de $5 \%{ }^{(29)}$.

A realização deste trabalho foi precedida pelo levantamento epidemiológico, aprovado pelo Comitê de Ética em Pesquisa da Faculdade de Ciências Farmacêuticas de Araraquara (Protocolo $n^{\circ}$ 09/2007).

\section{Resultados}

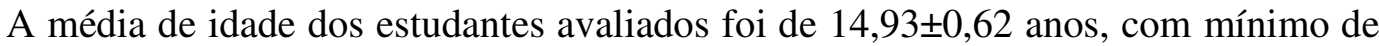
14 e máximo de 17 anos, sendo 51,06\% dos participantes do sexo feminino. Dos adolescentes, 65,96\% pertenciam às classes econômicas $\mathrm{A}$ e $\mathrm{B}, 33,33 \%$ à $\mathrm{C}$ e $0,71 \%$ à $\mathrm{D}$.

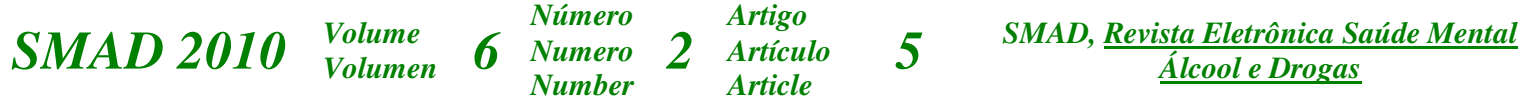



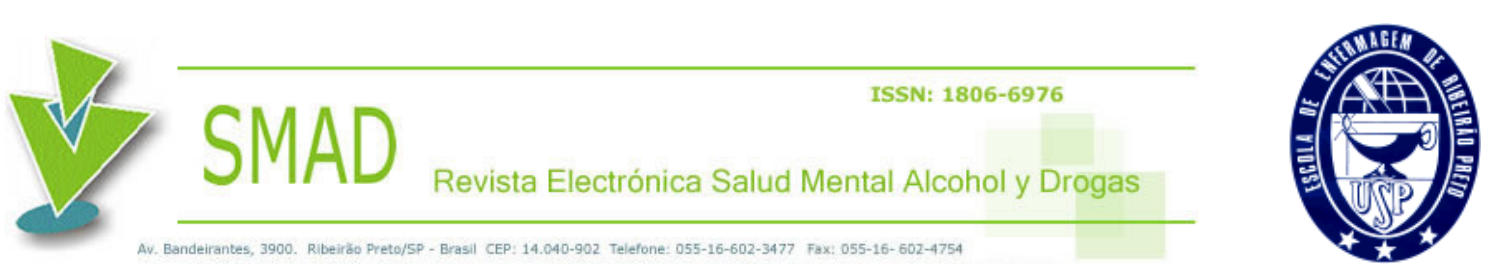

Quanto ao nível de escolaridade do chefe da família 2,17\% deles eram analfabetos, ou apresentavam primário incompleto, 17,39\% cursaram até a $4^{\text {a }}$ série do ensino fundamental, $21,01 \%$ possuíam ensino fundamental completo, $42,03 \%$ ensino médio completo e $17,39 \%$ superior completo. Cabe esclarecer que três estudantes não souberam informar o nível de escolaridade do chefe da família.

O consumo de bebidas alcoólicas pelos pais foi relatado por $58,87 \%$ dos participantes. A maioria dos estudantes afirmou possuir religião $(73,36 \%)$, praticar esportes $(68,97 \%)$, não trabalhar $(90,07 \%)$ e ter bom relacionamento familiar $(60,99 \%)$.

Pela pontuação total do AUDIT, 44 adolescentes $(31,21 \%)$ eram abstêmios, 93 $(65,96 \%)$ apresentavam comportamento de beber moderado, $3(2,13 \%)$ beber de risco e 1 $(0,71 \%)$ com uso nocivo de álcool.

Na Tabela 1 encontra-se o estudo da consistência interna do teste de identificação de transtornos devido ao álcool (AUDIT).

Tabela 1- Consistência interna do teste de identificação de transtornos devido ao uso de álcool (AUDIT), aplicado em adolescentes. Araraquara, 2009

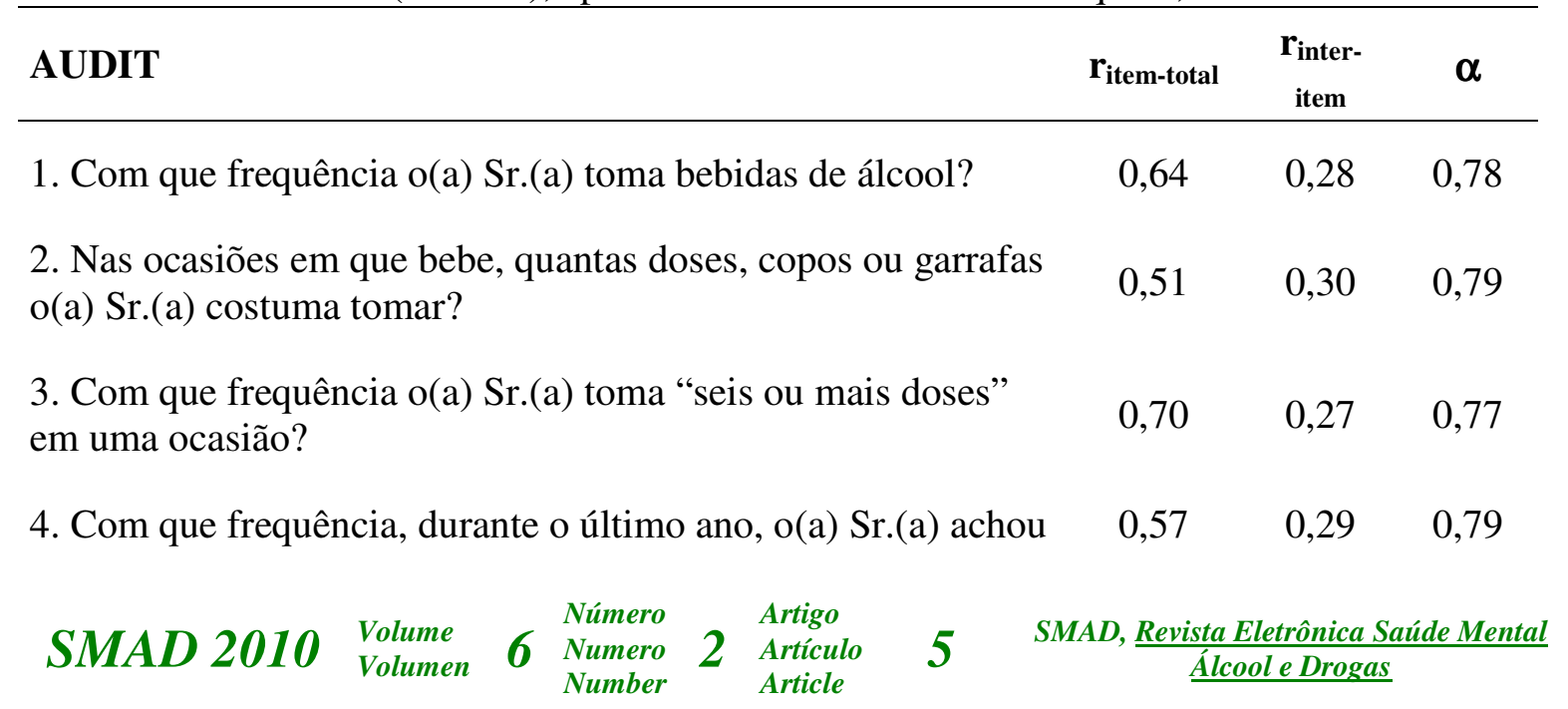


que não seria capaz de controlar a quantidade de bebida depois de começar?

5. Com que frequência, durante o último ano, o(a) Sr.(a) não conseguiu cumprir com algum compromisso por causa da $0,70 \quad 0,27 \quad 0,77$ bebida?

6. Com que frequência, durante o último ano, depois de ter bebido muito, o(a) Sr.(a) precisou beber pela manhã para se sentir melhor?

7. Com que frequência, durante o último ano, o(a) Sr.(a) sentiu culpa ou remorso depois de beber?

$0,77 \quad 0,26 \quad 0,76$

8. Com que frequência, durante o último ano, o(a) Sr.(a) não conseguiu se lembrar do que aconteceu na noite anterior por causa da bebida?

9. Alguma vez na vida o(a) Sr.(a) ou alguma outra pessoa já se machucou, se prejudicou por causa de o Sr.(a) ter bebido?

$0,72 \quad 0,27 \quad 0,77$

10. Alguma vez na vida algum parente, amigo, médico ou outro profissional da saúde já se preocupou com o(a) Sr.(a) por causa de bebida ou lhe disse para parar de beber?

Escala

$0,29 \quad 0,80$

Os resultados obtidos mostraram que o AUDIT apresentou excelente consistência interna $\left(\alpha=0,80 ; r_{\text {inter-item }}=0,29\right)$.

A estatística Kappa com ponderação linear $\left(\kappa_{\mathrm{p}}\right)$, por ponto e por intervalo de $95 \%$ de confiança $\left(\mathrm{IC}_{95 \%}\right)$, aplicada às questões do teste de identificação de transtornos devido ao álcool (AUDIT) e à classificação do risco de beber dos adolescentes, encontra-se na Tabela 2.

Tabela 2 - Reprodutibilidade do teste de identificação de transtornos devido ao uso de SMAD $2010 \begin{aligned} & \begin{array}{l}\text { Volume } \\ \text { Volumen }\end{array} \\ & \text { Sulumero }\end{aligned} \quad \begin{aligned} & \begin{array}{l}\text { Número } \\ \text { Number } \\ \text { Number }\end{array} \\ & 2\end{aligned} \begin{aligned} & \begin{array}{l}\text { Artigo } \\ \text { Artículo } \\ \text { Article }\end{array} \\ & \text { SMAD, Revista Eletrônica Saúde Mental }\end{aligned}$ 
álcool (AUDIT), aplicado em adolescentes. Araraquara, 2009

\section{AUDIT}

1. Com que frequência o(a) Sr.(a) toma bebidas de álcool?

2. Nas ocasiões em que bebe, quantas doses, copos ou garrafas o(a) Sr.(a) costuma tomar?

3. Com que frequência o(a) Sr.(a) toma "seis ou mais doses" em uma ocasião?

4. Com que frequência, durante o último ano, o(a) Sr.(a) achou que não seria capaz de controlar a quantidade de bebida depois de começar?

5. Com que frequência, durante o último ano, o(a) Sr.(a) não conseguiu cumprir com algum compromisso por causa da bebida?

6. Com que frequência, durante o último ano, depois de ter bebido muito, o(a) Sr.(a) precisou beber pela manhã para se sentir melhor?

7. Com que frequência, durante o último ano, o(a) Sr.(a) sentiu culpa ou remorso depois de beber?

8. Com que frequência, durante o último ano, o(a) Sr.(a) não conseguiu se lembrar do que aconteceu na noite anterior por causa da bebida?

9. Alguma vez na vida o(a) Sr.(a) ou alguma outra pessoa já se machucou, se prejudicou por causa de o Sr.(a) ter bebido?

10. Alguma vez na vida algum parente, amigo, médico ou outro profissional da saúde já se preocupou com o(a) Sr.(a) por causa de bebida ou lhe disse para parar de beber? $\kappa_{\mathbf{p}} \quad \mathrm{IC}_{\mathbf{9 5} \%} \quad$ Classificação

0,82 0,73-0,91 Ótima

$0,73 \quad 0,58-0,88 \quad$ Boa

$0,71 \quad 0,55-0,86 \quad$ Boa

$0,75 \quad 0,53-0,96 \quad$ Boa

$0,83 \quad 0,51-1,00 \quad$ Ótima

0,66 0,22-1,00 Boa

0,66 0,31-1,00 Boa

$0,81 \quad 0,66-0,96 \quad$ Ótima

0,65 0,37-0,79 Boa

$\begin{array}{llll}\text { Classificação de risco } & 0,75 & 0,56-0,94 & \text { Boa }\end{array}$

SMAD $2010 \begin{aligned} & \text { Volume } \\ & \text { Volumen }\end{aligned} \quad 6 \begin{aligned} & \begin{array}{l}\text { Número } \\ \text { Numero } \\ \text { Number }\end{array} \\ & \text { Surticule }\end{aligned} \begin{aligned} & \begin{array}{l}\text { Artigo } \\ \text { Artículo } \\ \text { Article }\end{array} \\ & \text { SMAD, Revista Eletrônica Saúde Mental }\end{aligned}$ 

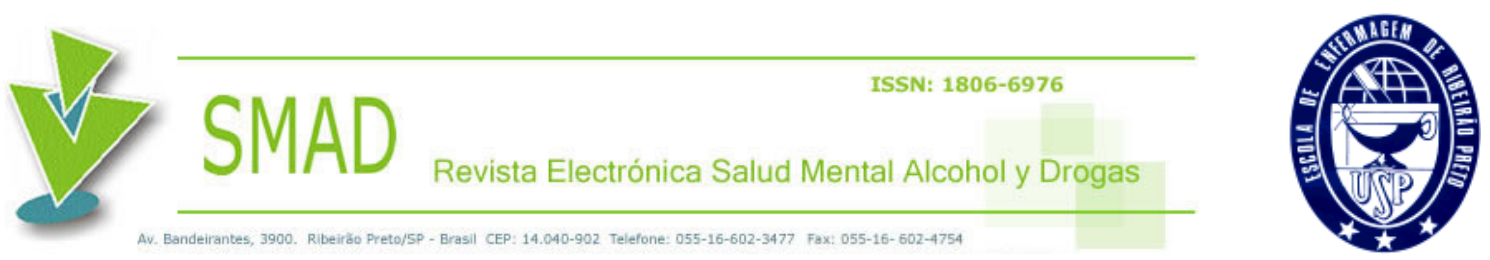

De acordo com os resultados, as questões referentes à frequência de consumo de bebidas alcoólicas $\left(\kappa_{\mathrm{p}}=0,82 ; \quad \mathrm{IC}_{95 \%}: 0,73-0,91\right)$, incapacidade de cumprir algum compromisso $\left(\kappa_{\mathrm{p}}=0,83, \mathrm{IC}_{95 \%}: 0,51-1,00\right)$ e de lembrar do que aconteceu na noite anterior por causa da bebida $\left(\kappa_{\mathrm{p}}=0,81, \mathrm{IC}_{95 \%}: 0,66-0,96\right)$ apresentaram ótima concordância, as demais questões apresentaram concordância classificada como "boa". Em relação à classificação do risco de beber dos estudantes, a reprodutibilidade também foi "boa" $\left(\kappa_{\mathrm{p}}=0,75, \mathrm{IC}_{95 \%}: 0,56-0,94\right)$.

\section{Discussão}

A necessidade da realização de estudos de confiabilidade dos instrumentos de pesquisa, relacionados ao consumo de álcool, tem sido de grande interesse devido à importância de se obter informações de qualidade adequada, tanto em contextos clínicos quanto epidemiológicos ${ }^{(13)}$.

Vários estudos nesse sentido são encontrados na literatura ${ }^{(12,16,18,20,30-35)}$, visando avaliar a confiabilidade do teste de identificação de transtornos devido ao álcool (AUDIT), porém, entre eles não se encontram trabalhos de consistência interna e reprodutibilidade do AUDIT, quando aplicado em adolescentes. Sendo assim, este estudo poderá representar contribuição importante ao agregar conhecimento sobre a confiabilidade do AUDIT, utilizado como instrumento de rastreamento de comportamento de risco de consumo de

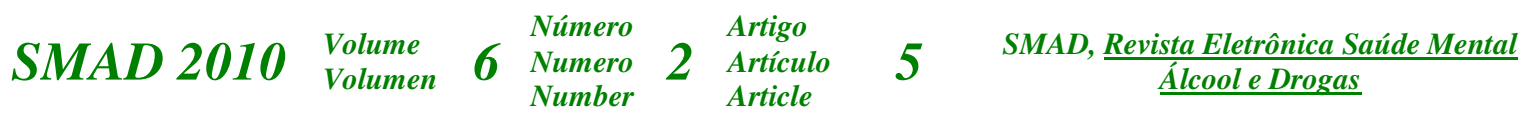



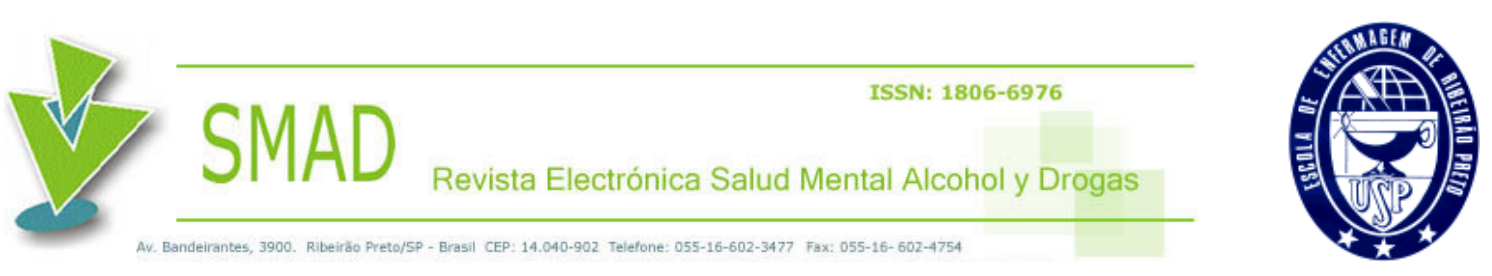

bebidas alcoólicas em adolescentes.

Em relação ao consumo de álcool, o presente estudo mostrou que 65,96\% dos jovens relataram consumir bebidas alcoólicas frequentemente, resultado esse, semelhante ao encontrado em estudo realizado em Cuiabá, MT, que apontou prevalência de $65,8 \%{ }^{(1)}$.

Estudos realizados no Brasil ${ }^{(36-38)}$, assim como em outros países ${ }^{(39-41)}$, têm mostrado que o álcool é a droga mais utilizada entre os adolescentes. Dados do CEBRID, obtidos por meio da realização de pesquisas com adolescentes, em dez cidades brasileiras, nos anos 1987, 1989 e 1997, revelam aumento do consumo de bebidas alcoólicas entre os jovens nos últimos $\operatorname{anos}^{(4)}$.

Quanto à confiabilidade, nota-se, na Tabela 1, que todas as questões do AUDIT apresentaram correlação interitem de $>0,20$ e que a escala obteve $\alpha=0,80$, resultados esses, satisfatórios, conforme aponta a literatura, onde coeficientes de correlação interitem acima de 0,20 são considerados adequados ${ }^{(42)}$ e coeficiente $\alpha$-Cronbach maior ou igual a 0,70 é considerado satisfatório ${ }^{(43)}$.

Assim, observou-se excelente consistência interna do AUDIT quando aplicado em adolescentes, mostrando resultado relevante, já que os estudos encontrados na literatura foram desenvolvidos em populações adultas. Em estudos, realizados em amostras de indivíduos adultos da Espanha e Nova Iorque, o AUDIT apresentou coeficiente $\alpha$-Cronbach de 0,93 e 0,85 , respectivamente ${ }^{(44-45)}$. Pesquisadores dos Estados Unidos e Coreia, ao avaliarem estudantes universitários, encontraram coeficiente $\alpha$-Cronbach de 0,94 e 0,85, respectivamente ${ }^{(46-47)}$.

SMAD $2010 \begin{aligned} & \begin{array}{l}\text { Volume } \\ \text { Volumen }\end{array} \\ & \text { Solumero }\end{aligned} \quad \begin{aligned} & \begin{array}{l}\text { Número } \\ \text { Number } \\ \text { Number }\end{array} \\ & 2\end{aligned} \begin{aligned} & \begin{array}{l}\text { Artigo } \\ \text { Artículo } \\ \text { Article }\end{array} \\ & \text { SMAD, Revista Eletrônica Saúde Mental }\end{aligned}$ 

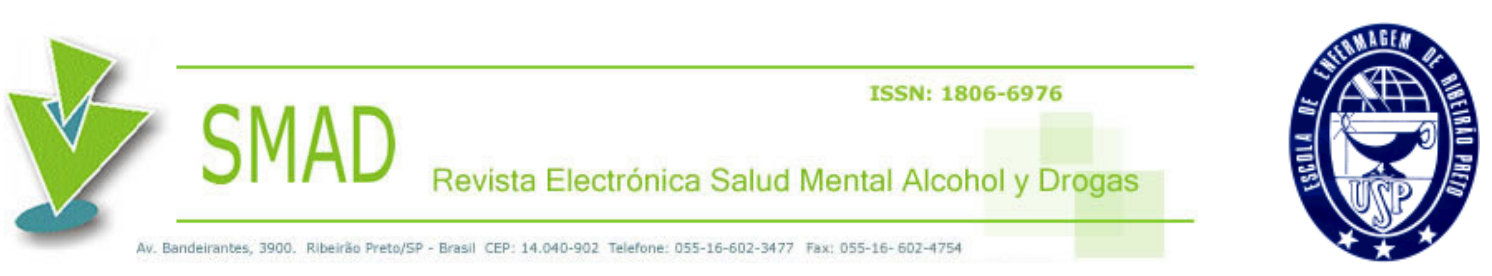

Em relação aos dados de reprodutibilidade do AUDIT do presente estudo, observase que os valores de Kappa variaram de 0,65 a 0,82, atestando concordância entre "boa" e “ótima”. Vários estudos chegaram a resultados semelhantes, onde pesquisadores ${ }^{(48)}$ da Nova Zelândia, ao avaliarem uma amostra de estudantes universitários, encontraram valor de Kappa de 0,92. Avaliando amostras de populações adultas, autores ${ }^{(31,35)}$ observaram reprodutibilidade de 0,92 e 0,84 , respectivamente, mostrando assim resultados satisfatórios quanto à reprodutibilidade do instrumento utilizado.

Os achados de confiabilidade do presente estudo atestam que o AUDIT pode ser um instrumento de rastreamento confiável para aplicação em adolescentes, entretanto, sua comparação com outros estudos ainda é difícil, devido à escassez de pesquisas com escolares nessa faixa etária.

Outro aspecto a ser ressaltado é o cuidado metodológico que os pesquisadores devem observar na abordagem dos adolescentes, uma vez que é fundamental despertar o interesse dos jovens para participarem do estudo de maneira séria e comprometida, visando evitar o levantamento de informações distorcidas. Assim, deve-se enfatizar a importância das visitas de esclarecimento, utilizando linguagem acessível, realizadas com o grupo antes da aplicação do AUDIT.

Pesquisadores $^{(49-50)}$ afirmam que profissionais envolvidos com programas de atenção à saúde, educação social e orientações a adolescentes apresentam dificuldades de abordagem desse grupo etário, devido, principalmente, à desmotivação, mal-estar e até mesmo irritação típica entre os adolescentes, durante o cumprimento de atividades SMAD $2010 \begin{aligned} & \begin{array}{l}\text { Volume } \\ \text { Volumen }\end{array} \\ & \text { Sulumero }\end{aligned} \quad \begin{aligned} & \begin{array}{l}\text { Número } \\ \text { Number } \\ \text { Number }\end{array} \\ & 2\end{aligned} \begin{aligned} & \begin{array}{l}\text { Artigo } \\ \text { Artículo } \\ \text { Article }\end{array} \\ & \text { SMAD, Revista Eletrônica Saúde Mental }\end{aligned}$ 
programadas. Os autores ressaltam que para desenvolver atividades com adolescentes, os profissionais devem estar capacitados e possuir habilidades de comunicação para o sucesso dessa abordagem.

\section{Conclusão}

O AUDIT apresentou confiabilidade satisfatória quando aplicado em adolescentes e com base em suas características de autopreenchimento, fácil compreensão e aplicação. Pode ser indicado como instrumento de rastreamento em estudos epidemiológicos, visando analisar o padrão de uso do álcool entre adolescentes.

\section{Referências}

1. Souza DPO, Areco KN, Silveira DX Filho. Álcool e alcoolismo entre adolescentes da rede estadual de ensino de Cuiabá, Mato Grosso. Rev Saude Publica. 2005;39(4):585-92.

2. Pechansky F, Szobot CM, Scivoletto, S. Uso de álcool entre adolescentes: conceitos, características epidemiológicas e fatores etiopatogênicos. Rev Bras Psiquiatr. 2004;26(1):14-7.

3. Pechansky F, Barros F. Problems related to alcohol consumption by adolescents living in the city of Porto Alegre, Brazil. J Drug Issues. 1995;25(4):735-50.

4. Galduróz JCF, Noto AR, Fonseca AM, Carlini EA. V Levantamento nacional sobre o SMAD $2010 \begin{aligned} & \begin{array}{l}\text { Volume } \\ \text { Volumen }\end{array} \\ & \text { SM }\end{aligned} \begin{aligned} & \begin{array}{l}\text { Número } \\ \text { Numero } \\ \text { Number }\end{array} \\ & 2\end{aligned} \quad \begin{aligned} & \begin{array}{l}\text { Artigo } \\ \text { Artículo } \\ \text { Article }\end{array} \\ & \text { SMAD, Revista Eletrônica Saúde Mental }\end{aligned}$ 

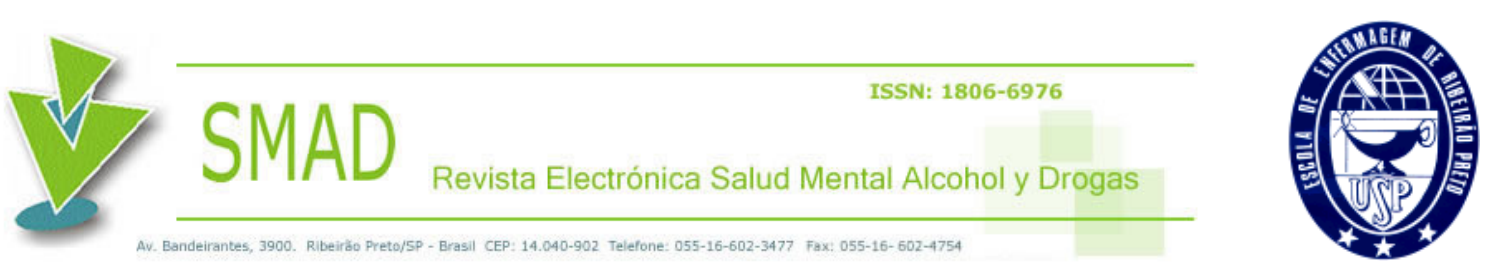

consumo de drogas psicotrópicas entre estudantes do ensino fundamental e médio da rede pública de ensino nas 27 capitais brasileiras: 2004. São Paulo: Centro Brasileiro de Informações sobre Drogas Psicotrópicas (CEBRID); UNIFESP; 2005.

5. Babor TF, Higgins-Biddle JC, Saunders JB. The alcohol use disorder identification test: guideline for use in primary care. Geneva: WHO-World Health Organization, Department of Mental Health and Substance Dependence; 2001.

6. Babor TF, Higgins-Biddle JC. Brief intervention for hazardous and harmful drinking: a manual for use in primary care. Geneva: WHO-World Health Organization; 2001.

7. Saunders JB, Aasland OG, Amundsen A, Grant M. Alcohol consumption and related problems among primary health care patients: WHO collaborative project on early detection of persons with harmful alcohol consumption I. Addiction. 1993;88(2):349-62.

8. Saunders JB, Aasland OG, Babor TF, Fuente JR, Grant M. Development of the alcohol use disorders identification test (AUDIT): WHO collaborative project on early detection of persons with harmful alcohol consumption II. Addiction. 1993;88(6):791-804.

9. Allen JP, Litten RZ, Fertig JB, Babor T. A review of research on the alcohol use disorders identification test (AUDIT). Alcohol Clin Exp Res. 1997;21(4):613-9.

10. Fleming MF, Barry KL, Macdonald R. The alcohol use disorders identification test (AUDIT) in a college sample. Int J Addict. 1991; 26:1173-85.

11. Claussen B, Aasland OG. The alcohol use Disorders Identification Test (AUDIT) in a routine health examination of long-term unemployed. Addiction 1993; 88(2):363-8.

12. Isaacson JH, Butler R, Zacharek M, Tzelepis A. Screening with the Alcohol Use

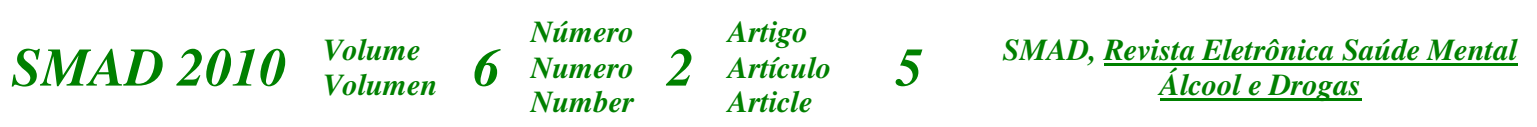



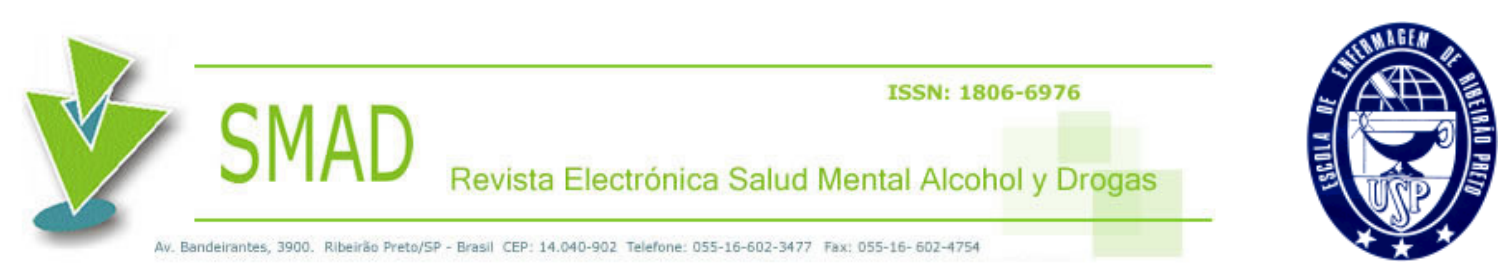

Disorders Identification Test (AUDIT) in an inner-city population. J Gen Intern Med 1994; 9:550-3.

13. Powell JE, Mclnness E. Alcohol use among older hospital patients: findings from an Australian study. Drug Alcohol Rev. 1994;13(1):5-12.

14. Bohn MJ, Babor TF, Kranzler HR. The Alcohol Use Disorders Identification Test (AUDIT): validation of a screening instrument for use in medical settings. J Stud Alcohol. 1995;56(4):423-32.

15. Cherpitel CJ. Analysis of cut points for screening instruments for alcohol problems in the emergency room. J Stud Alcohol. 1995;56(6):695-700.

16. Conigrave KM, Saunders JB, Reznik RB. Predictive capacity of the AUDIT questionnaire for alcohol-related harm. Addiction. 1995;90:1479-85.

17. Rigmaiden RS, Pistorello J, Johnson J, Mar D, Veach TL. Addiction medicine in ambulatory care: prevalence patterns in internal medicine. J Subst Abuse. 1995;16(1):4957.

18. Piccinelli M, Tassari E, Bortolomasi M, Piasere O, Semenzin M, Garzotto N, et al. Efficacy of the Alcohol Use Disorders Identification Test (AUDIT) as a screening tool for hazardous alcohol intake and related disorders in primary care: A validity study. BMJ. $1997 ; 314: 420-4$.

19. Skipsey K, Burleson JA, Kranzler HR. Utility of the AUDIT for the identification of hazardous or harmful drinking in drug-dependent patients. Drug Alcohol Depend. 1997; 45(1):157-63.

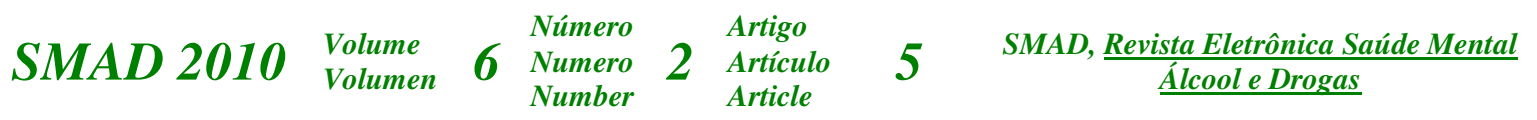



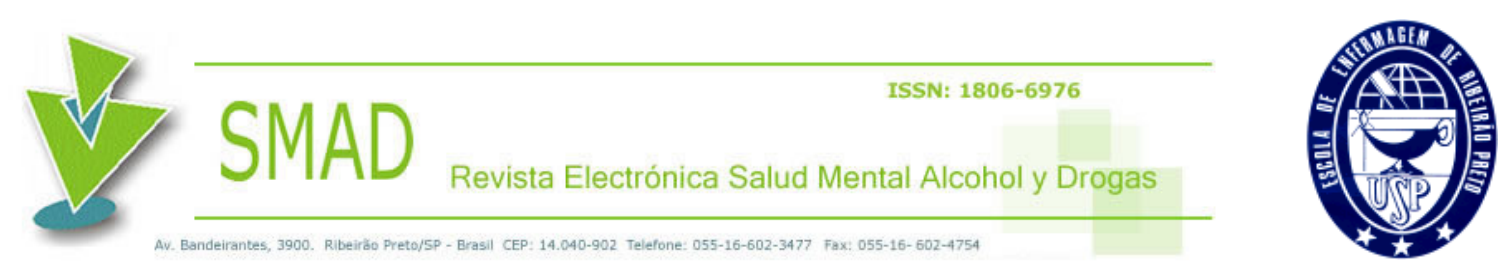

20. Volk RJ, Steinbauer JR, Cantor SB, Holzer CE. The Alcohol Use Disorders Identification Test (AUDIT) as a screen for at-risk drinking in primary care patients of different racial/ ethnic backgrounds. Addiction. 1997;92:197-206.

21. Fiellin DA, Carrington RM, O'Connor, PG. Screening for alcohol problems in primary care: a systematic review. Arch Intern Med. 2000;160:1977-89.

22. Marsh A, Smith L, Saunders B, Piek J. The impaired control scale: confirmation of factor structure and psychometric properties for social drinkers and drinkers in alcohol treatment. Addiction. 2002;97:1339-46.

23. Donovan DM, Kivlahan DR, Doyle SR, Longabaugh R Greenfield SF. Concurrent validity of the Alcohol Use Disorders Identification Test (AUDIT) and AUDIT zones in defining levels of severity among out-patients with alcohol dependence in the COMBINE study. Addiction. 2006; 101:1696-1704.

24. ABEP - Associação Brasileira de Empresas de Pesquisa. Critério de Classificação Econômica Brasil; 2008 [acesso em: 7 mar 2008]. Disponível em: http://www.abep.org/codigosguias/Critério_Brasil_2008.pdf

25. Mendéz EB. Uma versão brasileira do AUDIT (Alcohol Use Disorders Identification Test) [dissertação]. Pelotas (RS): Universidade Federal de Pelotas; 1999.

26. Lima CT, Freire ACC, Silva APB, Teixeira RM, Farrel M, Prince M. Concurrent and construct validity of the AUDIT in an urban Brazilian sample. Alcohol Alcohol. 2005;40(6):584.

27. Cronbach LJ. Coefficient alpha and the internal structure of the tests. Psychology. 1951;

SMAD $2010 \begin{aligned} & \text { Volume } \\ & \text { Volumen }\end{aligned} \quad 6 \begin{aligned} & \begin{array}{l}\text { Número } \\ \text { Numero } \\ \text { Number }\end{array} \\ & \text { Sulticule }\end{aligned} \quad \begin{aligned} & \begin{array}{l}\text { Artigo } \\ \text { Artículo } \\ \text { Article }\end{array} \\ & \text { SMAD, Revista Eletrônica Saúde Mental }\end{aligned}$ 

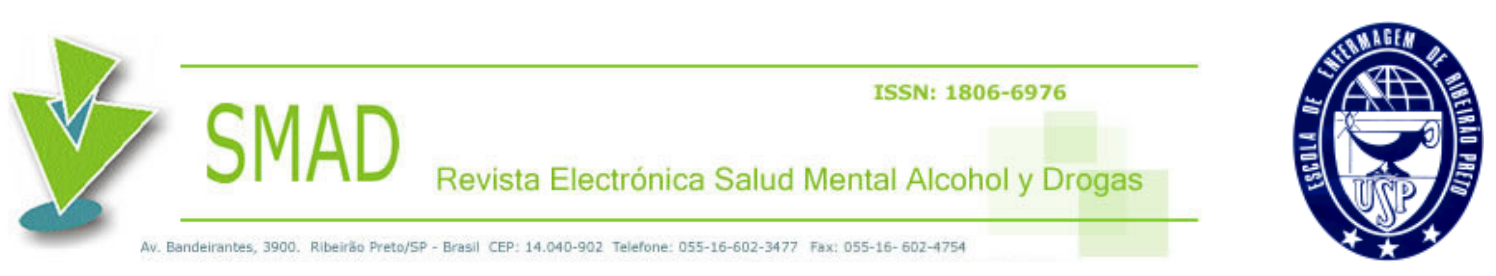

$16: 297-334$

28. Cronbach LJ. My current thoughts on coefficient alpha and successor procedures. CSE Report 643. Stanford: Stanford University; 2004. 32 p.

29. Landis JR, Koch GG. The measurement of observer agreement for categorical data. Biometrics. 1977;33(1):159-74.

30. Bradley KA, Mcdonnel MB, Bush K, Kivilahan DR, Diehr P, Fihn SD. The AUDIT alcohol consumption questions: reliability, validity, and responsiveness to change in older male primary care patients. Alcohol Clin Exp Res. 1998;22:1842-9.

31. Daeppen JB, Yersin B, Landry U, Pécoud A, Decrey H. Reliability and validity of the Alcohol Use Disorders Identification Test (AUDIT) imbedded within a general health risk screening questionnaire: results of a survey in 322 primary care patients. Alcohol Clin Exp Res. 2000;24(5):659-65.

32. Medina-Mora E, Carreno S, Fuente JR. Experience with the alcohol use disorders identification test (AUDIT) in Mexico. Recent Dev Alcohol. 1998;14(1):383-96.

33. Rubio VG, Bermejo VJ, Caballero SSMC, Santo-Domingo CJ. Validation of the Alcohol Use Disorders Identification Test (AUDIT) in primary care. Rev Clin Esp. 1998;198:4-11.

34. Schmidt A, Barry KL, Fleming MF. Detection of problem drinkers: the Alcohol Use Disorders Identification Test (AUDIT). South Med J. 1995;88:52-9.

35. Selin KH. Test- Retest Reliability of the Alcohol Use Disorder Identification Test in a General Population Sample. Alcohol Clin Exp Res. 2003;27(9):1428-35.

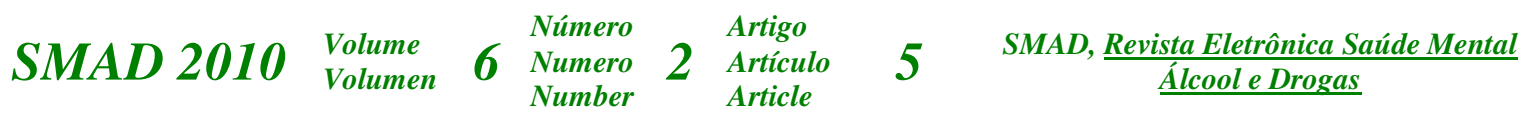


36. Deitos FT, Santos RP, Pasqualotto AC, Segat FM, Guillande S, Benvegnú LA. Prevalência do consumo de tabaco, álcool e drogas ilícitas em estudantes de uma cidade de médio porte no sul do Brasil. Inf Psiquiatr. 1998;17:11-6.

37. Galduróz, JCF, Noto AR, Carlini EA. IV Levantamento sobre o uso de drogas entre estudantes de $1^{\circ}$ e $2^{\circ}$ graus em 10 capitais brasileiras - 1997. São Paulo: Centro Brasileiro de Informações Sobre Drogas Psicotrópicas - CEBRID/ UNIFESP; 1997.

38. Muza GM, Bettiol H, Muccillo G, Barbieri MA. Consumo de substâncias psicoativas por adolescentes escolares de Ribeirão Preto, SP (Brasil). Rev Saude Publica. 1997;31:16370.

39. Aguilar HC, Martinez MRB. Consumo de alcohol y adolescencia. Rev Med Inst Mex Seguro Soc. 1993;31:279-81.

40. Patton GC, Hibbert M, Rosier MJ, Carlin JB, Caust J, Bowes G. Patterns of common drug use in teenagers. Aust J Public Health. 1995; 19:393-9.

41. Pecci MC. Varones jóvenes y sustancias psicoativas. Acta Psiquiatr Psicol Am Lat. $1995 ; 41: 288-99$.

42. Streiner D, Noeman G. Health measurement scales. A practical guide to their development and use. Oxford: Oxford University Press; 1995.

43. Nunnally JC, Bernstein IH. Psychometric theory. $3^{\text {rd }}$ ed. New York: WCB/Mc Graw Hill; 1994.

44. Torres, LAP, García JAF, Vega RA, Palomino MM, Rebollo EM, Moral RR. Validación del cuestionario AUDIT para la identificación del consumo de riesgo y de los

SMAD $2010 \begin{aligned} & \text { Volume } \\ & \text { Volumen }\end{aligned} \quad 6 \begin{aligned} & \begin{array}{l}\text { Número } \\ \text { Numero } \\ \text { Number }\end{array} \\ & \text { Surticule }\end{aligned} \quad \begin{aligned} & \begin{array}{l}\text { Artigo } \\ \text { Artículo } \\ \text { Article }\end{array} \\ & \text { SMAD, Revista Eletrônica Saúde Mental }\end{aligned}$ 
trastornos por el uso de alcohol em mujeres. Atenção Primaria. 2005;36(9):499-506.

45. Maisto SA, Conigliaro J, Mcneil M, Kraemer K, Kelley M. An Empirical Investigation of the Factor Structure of the AUDIT. Psychol Assess. 2000;12(3):346-53.

46. O'Hare T, Sherrer MV. Validating the Alcohol Use Disorder Identification Test With College First-Offenders. J Subst Abus Treat. 1999;17(1-2):113-9.

47. Chunn SS, Sohn AR. Correlates of problem drinking by the Alcohol Use Disorders Identification Test on Korean college campus. J Prev Med Pub Health. 2005;38:307-14.

48. Lennings CJ. Evaluation of the Leeds Dependence Questionnaire. J Child Adolesc Subst Abuse. 1999;8:73-87.

49. Abduch C. Grupos operativos com adolescentes. In: Schor N, Mota MSFT, Branco VC. Cadernos juventude, saúde e desenvolvimento. Brasília: Ministério da Saúde; 1999. p. 289-300. 50. Ruzany MH, Andrade CLT, Esteves MAP, Pina MF, Szwarcwald CL. Avaliação das condições de atendimento do Programa de Saúde do Adolescente no Município do Rio de Janeiro. Cad Saude Publica. 2002;18:639-49.

Recebido em: 01/02/2010

Aprovado em: 20/03/2010

\section{Como citar este artigo:}

Mattara FP, Ângelo PM, Faria JB, Campos JADB. Confiabilidade do teste de identificação de transtornos devido ao uso de álcool (AUDIT) em adolescentes. SMAD, Rev. Eletrônica Saúde Mental Álcool Drog. (Ed. port.) [Internet]. 2010 [acesso em: dia mês abreviado com ponto ano] ; 6(2):296-314. Disponível em: Endereço Eletrônico Visitado.

SMAD $2010 \begin{aligned} & \begin{array}{l}\text { Volume } \\ \text { Volumen }\end{array} \\ & \text { Solumero }\end{aligned} \quad \begin{aligned} & \begin{array}{l}\text { Número } \\ \text { Number } \\ \text { Number }\end{array} \\ & 2\end{aligned} \begin{aligned} & \begin{array}{l}\text { Artigo } \\ \text { Artículo } \\ \text { Article }\end{array} \\ & \text { SMAD, Revista Eletrônica Saúde Mental }\end{aligned}$ 